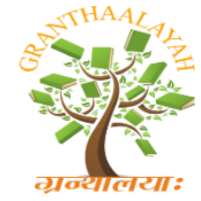

INTERNATIONAL JOURNAL OF RESEARCH GRANTHAALAYAH A knowledge Repository

RAST - 17

\title{
FABRICATION AND CHARACTERIZATION STUDIES OF LITHIUM- BORATE RUBY GLASSES CONTAINING GOLD NANOPARTICLES
}

\author{
Y. Shivaprakash ${ }^{* 1}$, R.V. Anavekar ${ }^{2}$ \\ ${ }^{* 1}$ Department of Physics, Governament First Grade College, Devanahalli, Bangalore, (R) \\ 521010, India \\ ${ }^{2}$ Department of Physics, Jnanabharathi Campus, Bangalore University, Bangalore-560 056, \\ Karnataka, India
}

DOI: https://doi.org/10.29121/granthaalayah.v5.i4RAST.2017.3307

\begin{abstract}
Glasses containing $\mathrm{Au}$ nanoparticles have been synthesized in the base glass of composition $30 \mathrm{Li}_{2} \mathrm{O}-70 \mathrm{~B}_{2} \mathrm{O}_{3}$ using gold chloride $\left(\mathrm{HAuCl}_{4} \cdot 3 \mathrm{H}_{2} \mathrm{O}\right)$ as a dopant. The characteristic ruby color has been observed which is attributed due to surface plasmon resonance of the Au nanoparticles. The hue of the color increases with the increase of concentration of the dopant. Presence of the gold nanoparticles has been confirmed by studying the optical absorption spectra in the visible range which show a characteristic absorption peak at $\sim 560 \mathrm{~nm}$. XRD and SEM studies show that the addition of $\mathrm{Au}$ increases the kinetics of growth and crystallization. ESR studies indicate that the electronic state of gold is either $\mathrm{Au}^{0}$ or $\mathrm{Au}^{+}$.
\end{abstract}

Keywords: Borate Glass; Gold Nanoparticles; Optical Absorption; SPR.

Cite This Article: Y. Shivaprakash, and R.V. Anavekar. (2017). "FABRICATION AND CHARACTERIZATION STUDIES OF LITHIUM-BORATE RUBY GLASSES CONTAINING GOLD NANOPARTICLES.” International Journal of Research - Granthaalayah, 5(4) RAST, 80-86. https://doi.org/10.29121/granthaalayah.v5.i4RAST.2017.3307.

\section{Introduction}

The formation of noble metal nanoparticles inside a glass has been extensively investigated for the past decades because of their excellent properties such as an ultrafast optical response and large third order non-linear susceptibility [1-4]. These kinds of glasses are expected to be promising materials for ultrafast optical switches and optical circuits with micrometer size. Hence, preparation and characterization of materials containing noble metal nanoparticles is the most challenging task for researchers in the area of materials science. Further, the fabrication of metal nanoparticles of different size and shape in glass matrix by various methods become the most advanced issue [3,4]. Glasses containing metal nanoparticles look very distinctive with different colors. Since the quantum size effects and enhanced surface effects are expected to 
influence the electronic and optical properties at much smaller particle sizes leads more interest for researchers to fabricate the metal nanoparticles in glasses [5,6]. One such investigation on gold ruby glass is due to their distinctive reddish ruby color. These glasses are mainly used not only for decorative glassware but also for many technological applications in the field of electrical and optical communications. Silicate ruby glasses were synthesized mainly by using $\mathrm{HAuCl}_{4} .3 \mathrm{H}_{2} \mathrm{O}$ along with reducing agent such as $\mathrm{Sn}$ based compounds and then annealing the glass for several hours at a particular temperature [7]. Metal nanoparticles in glasses are also precipitated using electron beam and laser irradiation. In these methods the metal ions are reduced but needs thermal treatment [8]. In recent years gold nanoparticles are also fabricated in certain germinate and borate glass matrix [9]. The conductivity studies have been examined as a function of frequency and temperature. Interestingly, here the mechanism of $\mathrm{dc}$ conduction or dipolar loss is quite different from ionic/electronic conducing glasses. Barium borate glasses with gold nanoparticles studies [2] show that these materials exhibit third order non-linearity.

In this communication, we report fabrication of gold nanoparticles in lithium-borate glass without thermal treatment and addition of any reducing agents. The samples have been characterized using X-ray diffraction, scanning electron microscopy (SEM), Electron spin resonance (ESR) and Optical absorption studies have been performed at UV-Visible range of wavelength.

\section{Experimental}

Borate glasses with the composition $30 \mathrm{Li}_{2} \mathrm{O}-70 \mathrm{~B}_{2} \mathrm{O}_{3}-\mathrm{x} \mathrm{HAuCl}_{4} 3 \mathrm{H}_{2} \mathrm{O}$ [LBA] (where $\mathrm{x}=0.01$, 0.04 and $0.06 \mathrm{wt} \%$ ) have been prepared by melt quenching technique. The powders were thoroughly mixed and then melted at $1000^{\circ} \mathrm{C}$ respectively. The melt was stirred well and kept again in the furnace at the melting temperature for about $10 \mathrm{~min}$. The melt was quenched between two brass plates at room temperature. The quenched glass samples so obtained were found to be ruby-red in color. The deepness of the color was found to be increasing with the increase in concentration of gold dopant. The samples were optically polished for optical measurements. Optical absorption spectra were recorded using UV-VIS Spectrometer [USB2000, Ocean Optics (USA)] in the visible range (400-1000 nm). For XRD (XPERT PRO, Philips), SEM [440I, LEO (U.K)] and ESR [Expands, Bruker (UK)] samples were powdered and examined at room temperature.

\section{Results and Discussion}

\section{Characterization by Optical Absorption}

The absorption spectra of the prepared glass $30 \mathrm{Li}_{2} \mathrm{O}-70 \mathrm{~B}_{2} \mathrm{O}_{3}[\mathrm{LB}]$ and respective glasses with gold nanoparticles (LBA) are shown in Fig. 1. 


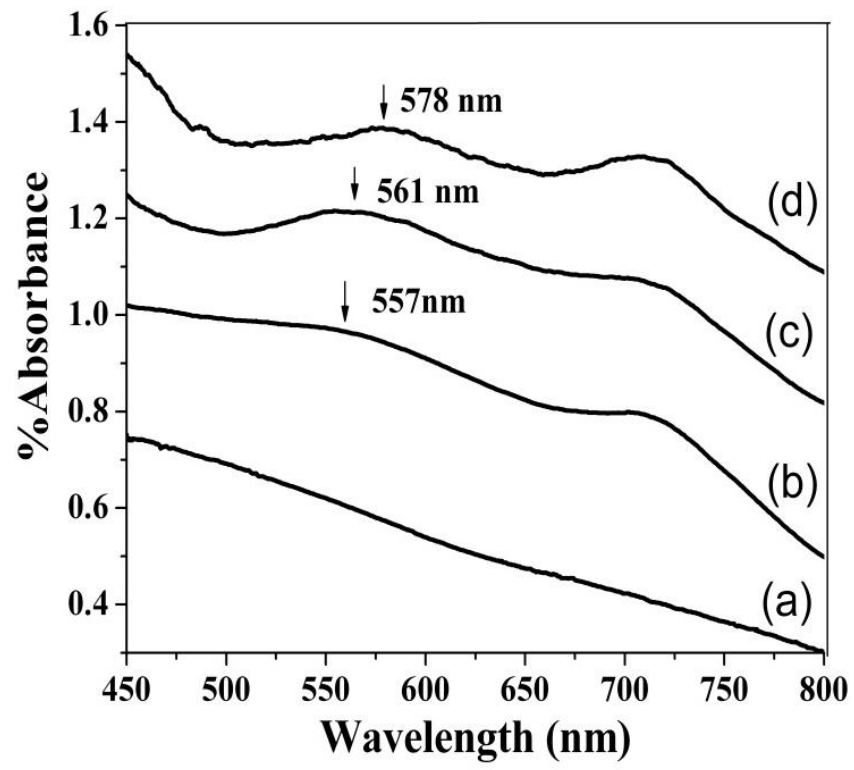

Figure 1: Absorption spectra of LB and LBA samples: (a) Sample without Au, (b) Sample with 0.01 wt\% of Au, (c) Sample with 0.04 wt \% of Au ,(d) Sample with 0.06 wt\% of Au.

It is clearly seen that, a broad absorption peak of each sample centered at 550-580 nm [Fig. 1 (bd)]. This peak is attributed to the Surface Plasmon Resonance (SPR) due to the metallic gold nanoparticles embedded in the glass matrix [10,11]. According to Mie theory [12], the mechanism of surface plasmon resonance is due to the polarization of the free electrons with respect to the much heavier ionic core of spherical gold nanoparticles in the presence of electric field of an incoming light wave. A net charge difference is felt at the nanoparticles boundaries which act as a restoring force and creates dipolar oscillation of the electrons with certain time period [5, 13-16]. As seen in the Fig. 1 (b-d), there is a slight shift in the peak position towards higher wavelength side which is attributed due to increase in the size of the gold nanoparticles with the increase of gold concentration [5]. There is no such absorption peak as seen in Fig 1(a). In addition to the peak $\sim 550 \mathrm{~nm}$, there is weak peak centered around $\sim 700 \mathrm{~nm}$ attributed to interband transition from the d-band to empty state in the conduction band of the metallic gold particles [6].

\section{Characterization by X-ray Diffraction (XRD)}

X-ray diffraction pattern of LB and LBA are shown in Fig. 2 in which one can clearly see the sharp peaks at $38.71^{\circ}, 44.87^{\circ}$ and $64.95^{\circ}$. These prominent peaks corresponds to reflections from the planes (111), (200) and (220) of gold respectively [3, 14, 16, 17]. It may be also noted that the intensities of these peaks increase with the increase of gold concentration. 


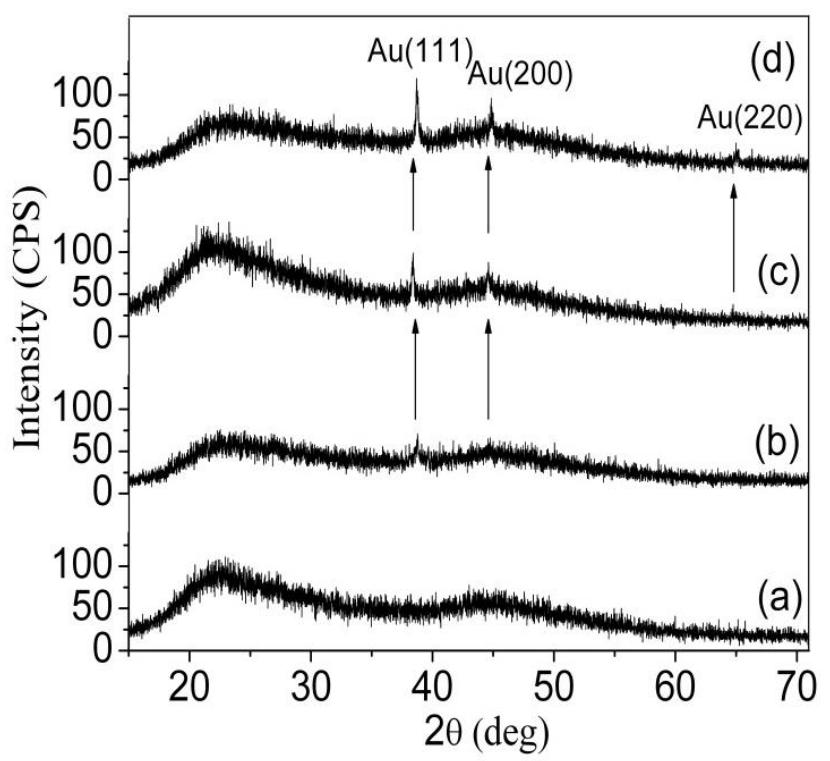

Figure 2: XRD Pattern of LB and LBA samples: (a) Sample without Au, (b) Sample with 0.01 wt $\%$ of $\mathrm{Au}$, (c) Sample with 0.04 wt \% of Au, (d) Sample with 0.06 wt\% of Au.

The average gold particle size in the LBA sample with $0.06 \mathrm{wt} \%$ of gold dopant was calculated using the standard Debye-Scherer equation mentioned below and foun $\mathrm{d}$ to be $\sim 28 \mathrm{~nm}$

$$
\mathrm{D}=\mathrm{K} \lambda / \beta \cos \theta
$$

where $\mathrm{K}$ is the particle shape factor (generally taken as 0.9 ), $\lambda$ is the wavelength of $\mathrm{CuK} \alpha$ radiation, $\beta$ is the integral breadth of a reflection (FWHM in radian), and $\theta$ is the Bragg angle at the peak position.

\section{Characterization by Scanning Electron Microscopy (SEM)}

SEM images of lithium-borate glass series are presented in Fig. 3 (a-d). One can notice in Fig. 3(a) that the image of the sample (LB) with $0 \%$ of gold dopant shows homogeneous phase, on the other hand the samples with different concentration of gold dopant (LBA) [Fig. 3(b) to 3(d)] clearly show the growth of small particles/crystallites of different size. It is extremely difficult to realize the presence of gold particles in the nano range from these images. However, one can conclude that the addition of gold dopant induces kinetics of the growth of particular phase seems to be layered structure in LBA samples. 


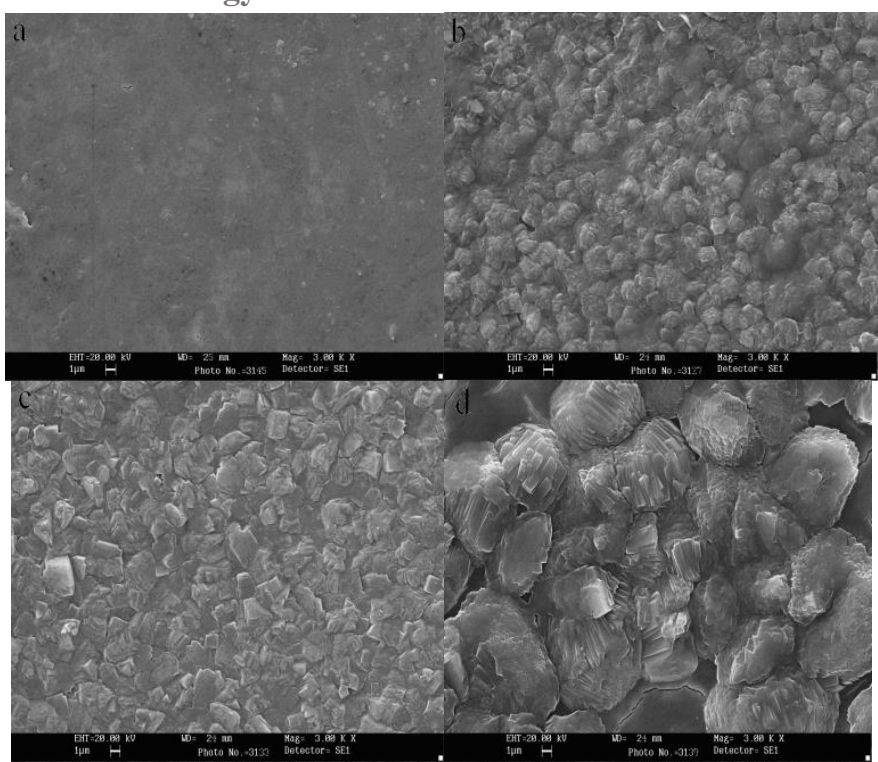

Figure 3: SEM records of LB and LBA samples: (a) Sample without Au, (b) Sample with wt\% of $\mathrm{Au}$, (c) Sample with 0.04 wt \% of Au, (d) Sample with $0.06 \mathrm{wt} \%$ of Au.

\section{Characterization by Electron Spin Resonance (ESR)}

Electron spin resonance (ESR) studies have been performed to understand the electronic state of the $\mathrm{Au}$ in LBA samples. It is well known that $\mathrm{Au}^{2+}$ are paramagnetic and ESR active. They can form several interesting complexes [18]. On the other hand, $\mathrm{Au}^{0}$ and $\mathrm{Au}^{+}$are ESR inactive [18, 19]. The ESR spectra of all the samples under investigation are shown in the Fig. 4.

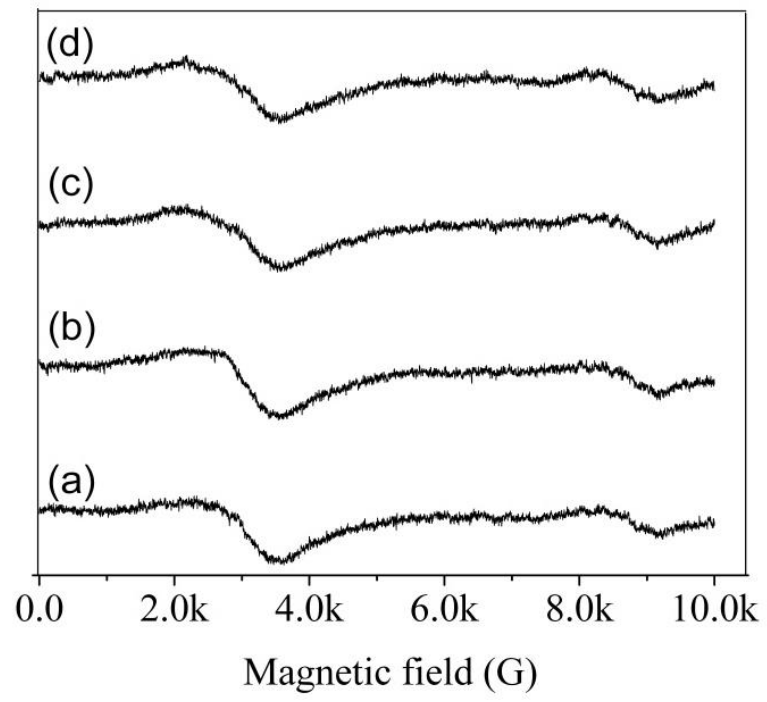

Figure 4: ESR spectrum of LB and LBA samples: (a) Sample without Au, (b) Sample with 0.01 wt $\%$ of Au, (c) Sample with 0.04 wt \% of Au, (d) Sample with 0.06 wt\% of Au.

It is clearly seen that there is neither sharp signal nor hyperfine split in the entire spectrum, which is indicating that the samples have neither paramagnetic ions nor unpaired electrons. Hence the electronic state of the gold must be either $\mathrm{Au}^{0}$ or $\mathrm{Au}^{+}[20]$. In a typical ruby glass, 
before striking the ruby color, gold is dissolved in $\mathrm{Au}^{+}$state. Further, a subsequent heat treatment (with the help of reducing species) gives the striking ruby color. In the present study, ruby color without reducing agent and heat treatment, the mechanism of $\mathrm{Au}^{+}$to $\mathrm{Au}^{0}$ appears to be complicated [4].

\section{Conclusions}

Gold nanoparticles have been fabricated in lithium borated glasses. The characteristic ruby color has been realized in all the glasses without adding any reducing agent and heat-treatment. Presence of gold nanoparticles has been confirmed by studying the optical absorption spectra. A characteristic peak obtained at $\sim 570 \mathrm{~nm}$ is attributed due to surface plasmon resonance. A morphological change due to the addition of gold dopant has been characterized by XRD and SEM techniques. XRD analysis of LBA glasses shows the reflection due to the presence of gold. SEM studies show that the addition of gold increases the kinetics of growth. ESR studies reveal that the electronic state of gold is either $\mathrm{Au}^{0}$ or $\mathrm{Au}^{+}$.

\section{Acknowledgements}

The authors thank the Department of Physics, Indian Institute of Science, Bangalore for providing the experimental facilities (XRD, ESR) and NAL, Bangalore for SEM.

\section{References}

[1] N.Venkataram, R. Sai Santosh Kumar, D. Narayana Rao, S.K. Medda, Sucheta De and Goutam de: J. Nanoscience and Nanotechnology, 8, 1990 (2006).

[2] Jun Sasia and Kazuyuki Hirao: J. App. Phy. 89(8), 4548 (2001).

[3] Jianqiang Hu, Zhouping Wang and Jinghoung Li: Sensors 7, 3299 (2007).

[4] Himanshu Jain, Ahmed Issa, Rajan V. Anavekar, Ronald Bohmer, Otmar Kanert and Reiner Kuchler: App. Phy. Lett. 95, 142908 (2009).

[5] Stephan Link and Mostafa A El-Sayed: Rev. Phy. Chem. 19(3), 409 (2000).

[6] B. Balamurugan and Toshiro Mayuyama: App. Phy. Lett. 87, 143105 (2005).

[7] S. Haslbeck, K.P. Martinek, L. Stievano and F.E.Wagner: Hyperfine Interact 165, 89 (2005).

[8] Jongho shin, Kyungsik Hang, Ki-Soo Lim, Ik-Bu Sohn, Young-Chul Noh and Jongmin Lee:Appl. Phys. A, 93, 923 (2008).

[9] Ahmed Issa, Rajan. V. Anavekar, Reiner Kuchler, Rolan Bohmer, Otmar Kanert and Himanshu Jain: Z. Phys. Chem. 223, 1273 (2009).

[10] Collen L. Nehl and Jaspn H. Hafner: J. Mat. Chem. 18, 2415 (2008).

[11] Jeremy Vosburgh, Robert H. Doremus: J. Non-Cryst. Solids 349, 309 (2004).

[12] G. Mie: Beiträge zur Optik trüber Medien, speziell kolloidaler Metallösungen: Ann. d Pysik Vierte Folge, Band 25(3), 377 (1908).

[13] Hongbo Liao, Weixinn Lu, Shengwen Yu, Weijja Wen and George K.L.Wang: Opt. Soc. Am. B 22(9), 1923 (2005).

[14] Kensuke Akamatsu and Shigehito Deki: J. Mat. Chem. 7(9), 1773 (1997).

[15] Cleverland Engene Rayford II, George Shehatz, and Kevin Shufor: Spring-2005 Nanoscape 2(1), 27 (2005).

[16] Xiaogang Liu, Mianqiang Wu, Benjamin H.Wunsch, Robert J., Barscotti Jr and Francesco Stellacci: Small 2(8-9), 1046 (2006).

[17] Ki-Chul Kim, Im-Bok Lee, Dae-Joon Kemg and Sunglyul Maeng: ETRI Journal 29(6), 814 (2007). 
[Shivaprakash et. al., Vol.5 (Iss.4: RAST), April, 2017]

ICV (Index Copernicus Value) 2015: 71.21

Recent Advances in Science \& Technology
ISSN- 2350-0530(O), ISSN- 2394-3629(P)

IF : 4.321 (CosmosImpactFactor), 2.532 (I2OR)

InfoBase Index IBI Factor 3.86

[18] Z. Qu and E. Roduner: In situ ESR study of gold supported on NaY zeolite: J. Chem. Eng. 4, 602 (2009).

[19] T. Vanngard, S. Akerstrom: Nature 184, 183 (1959).

[20] J.W Orton: Electron Paramagnetic Resonance "An Introduction to transition group ions in crystals": ILIFFE BOOKS LTD. LONDON. 1968.

*Corresponding author.

E-mail address: yshivaprakash@gmail.com 